\title{
Position-Sensitive STEM Detectors for High-Sensitivity Phase Detection
}

\author{
Timothy J. Pennycook ${ }^{1,2}$, Lewys Jones ${ }^{1}$ and Peter D. Nellist ${ }^{1,2}$
}

1. Department of Materials, University of Oxford, Parks Road, Oxford OX1 3PH, UK

2. SuperSTEM Laboratory, Daresbury, WA4 4AD, UK

The most commonly-used mode of imaging in the scanning transmission electron microscope (STEM) has made use of the annular dark-field (ADF) detector. Recently, however, there has been increased interest in bright-field (BF) modes. By reciprocity, a small BF detector with collection angles that are about an order of magnitude smaller than the convergence angle of the probe (see Fig. 1) provides an imaging mode that is equivalent to phase contrast imaging in the conventional high-resolution transmission electron microscope (HRTEM). A small detector is necessary to maintain sufficient coherence for efficient phase contrast, but leads to low efficiency of use of incident electrons in the STEM configuration because most of the incident electrons are not collected.

The annular bright-field (ABF) mode of imaging has been reevaluated recently for the purpose of atomic resolution imaging in instruments fitted with spherical aberration correctors. This mode has been successful in demonstrating imaging of columns of low-Z elements including hydrogen. Contrast in the $\mathrm{ABF}$ image may be generated through channelling effects, or through a phase-contrast effect if residual aberrations are present, leading to some complications in interpretation [1].

An alternative approach is to use multiple detectors in the STEM to record several images simultaneously that can then be reconstructed into one or more images. An example of this approach is the use of 4 detectors in a quadrant to give a differential phase contrast (DPC) image [2]. The logical extension of this approach is to use a pixellated detector to record the entire bright-field disc in the STEM detector plane. Using such an approach Kimoto and Ishizuka [3] have shown that differential phase contrast effects may be seen. Earlier, however, Rodenburg et al [4] demonstrated a method by which the entire data set could be used to retrieve the phase of the object function and extend the resolution of the microscope.

The work by Rodenburg et al was focused on overcoming the diffraction limit to imaging resolution. Here we re-evaluate the technique with regard to the phase sensitivity of the approach. Samples of soft materials often consist of low-Z elements and are radiation sensitive. For single electron scattering, only the phase of the electron wave is modified, and so it is desireable to detect that phase shift with the highest possible signal to noise ratio for the lowest possible dose.

Figure 2 shows preliminary data from $\mathrm{SrTiO}_{3}$ where both an $\mathrm{ABF}$ and DPC signal have been extracted from the same series of CCD images. These images were recorded at a subset of the probe positions used to create an ADF image, which is shown both at full resolution and subsampled to just the points at which the CCD images were aquired. Line profiles of the regions indicated by arrows in the ABF, DPC and subsampled ADF images are shown in Fig. 2 B. In the middle of the profile is a Sr column, well resolved in the ADF image. The contrast in the ABF signal is seen to be considerably higher than the DPC signal. The DPC signal was constructed from two quadrants or half of the BF disk, while the ABF image was constructed with an inner angle $50 \%$ of the outer angle of the BF disk and therefore used $3 / 4$ 
of the $\mathrm{BF}$ disk. The strength of the $\mathrm{ABF}$ signal over the DPC signal is therefore considerably greater than the extra proportion of the BF disk used to construct it.

We will present further experimental developments, data and theoretical calculations comparing various $\mathrm{BF}$ modes to investigate the optimal configuration for the maximum phase sensitivity with the lowest dose. Such data can be compared with data recorded simultaneously using an ADF detector, allowing low-spatial frequency data to also be included [5].

\section{References:}

[1] S. Lee et al. Ultramicroscopy, 125, (2013), p. 43.

[2] N. Shibata et al, Nature Physics, 8, (2012), 611.

[3] K. Kimoto and K. Ishizuka, Ultramicroscopy, 111, (2011), p. 1111.

[4] J. M. Rodenburg, B. C. McCallum and P. D. Nellist, Ultramicroscopy, 48, (1993), p. 303.

[5] This research was supported by the EPSRC through the UK National Facility for AberrationCorrected STEM (SuperSTEM)
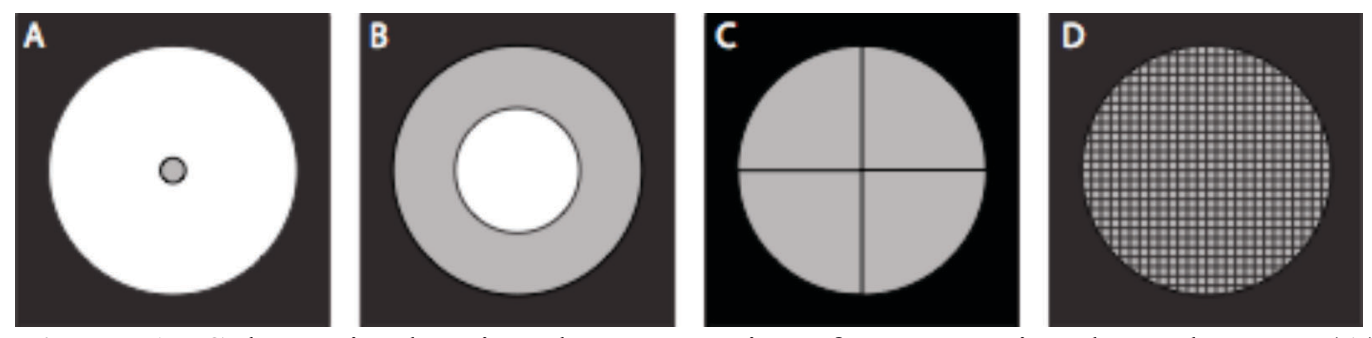

Figure 1. Schematic showing the geometries of a conventional BF detector (A), an ABF detector (B), the quadrant detectors used in DPC (C), and recording the entire BF disk with a pixelated detector (D). The convergence angle is indicated by the inner bound of the black outer region. Detectors are colored grey with black edges.
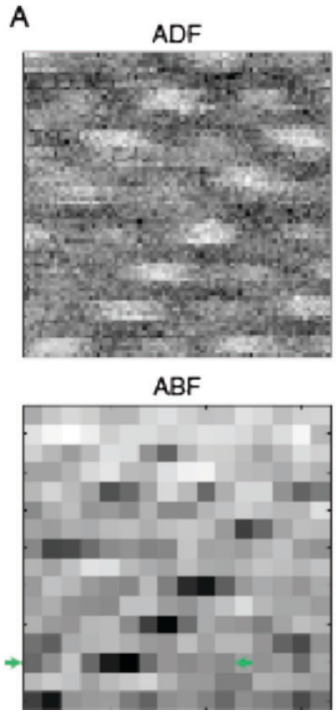

Subsampled ADF

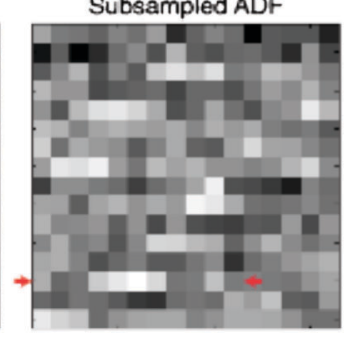

DPC

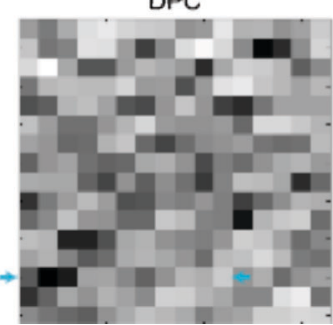

B

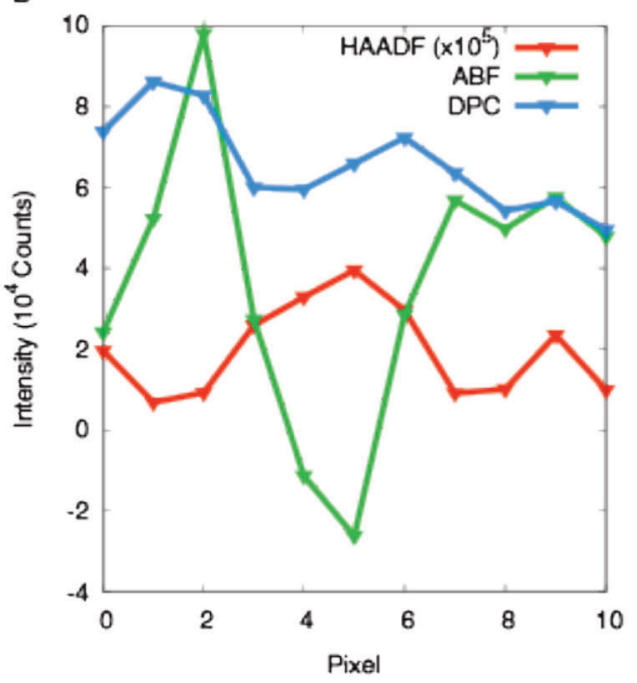

Figure 2. Preliminary $\mathrm{ABF}$ and $\mathrm{DPC}$ data extracted from the same series of $\mathrm{CCD}$ images of the bright field disk recorded in parallel with an ADF image. Arrows in A indicate the pixels profiled in B. 